\title{
Sebaceous hyperplasia: systemic treatment with isotretinoin ${ }^{*}$
}

\author{
Sandra Tagliolatto ${ }^{1}$ \\ Maurício Mota de Avelar Alchorne ${ }^{3}$
}

\author{
Octavio de Oliveira Santos Neto ${ }^{2}$ \\ Mauro Yoshiaki Enokihara ${ }^{3}$
}

DOI: http://dx.doi.org/10.1590/abd1806-4841.20153192

\begin{abstract}
The study aimed to verify the therapeutic action of isotretinoin in the treatment of sebaceous hyperplasia. During two months, 20 patients with sebaceous hyperplasia took isotretinoin at a dosage of $1 \mathrm{mg} / \mathrm{kg}$ per day. Their skin lesions were counted and photographed before and after treatment and re-evaluated two years later. The average number of sebaceous hyperplasia lesions before treatment was 24 per patient. At the end of two months of therapy, the number of lesions decreased to 2 per patient. The statistically analyzed data showed a reduction in the number of lesions following isotretinoin use $(p<0.05)$. Two years after the end of the treatment, the average number of sebaceous hyperplasia lesions was 4 per patient. There were no severe side effects. Thus, the data analysis suggests that isotretinoin is a safe and effective drug for treating the disease under study.
\end{abstract}

Keywords: Clinical trial; Isotretinoin; Sebaceous Gland Diseases; Therapies, investigational

\section{INTRODUCTION}

Sebaceous hyperplasia is generally treated using surgical methods, including cauterizations and excisions. However, depending on the number of lesions and the cicatricial response of the skin, it may be necessary to avoid such interventions. ${ }^{1}$ Alternatives, including lasers and other therapeutic methods like photodynamic therapy, can be costly and difficult to access. ${ }^{2,3}$

Drawing on literature reports about the use of isotretinoin in isolated cases of sebaceous hyperplasia, as well as studies that demonstrate histopathologically the reduction of the sebaceous gland through use of systemic isotretinoin, and by reviewng studies on the sebo-supressive action of isotretinoin, we assumed the drug's effectiveness in treating sebaceous gland hyperplasia. ${ }^{4-6}$

The use of isotretinoin in treating sebaceous hyperplasia is justified by its effectiveness in reducing the size of the sebaceous gland, in addition to its action in diminishing the proliferation of basal sebocytes, suppressing the production of sebum and inhibiting the differentiation of the sebocytes in vivo. ${ }^{5}$

Several papers have studied the mechanism of the retinoids' anti-sebotropic effect. Some research lines attempt to explain its sebo-supressive action by studying the apoptosis of the sebocytes, while others hold that the answer lies in the interaction of isotretinoin in the metabolism of the androgens. ${ }^{7.8}$

Although the molecular base for the retinoids' anti-sebotropic activity has not been explained entirely, due to disagreement as to whether the mechanism depends on nuclear receptors, many laboratory studies of sebocytes and human sebaceous glands confirm the effective action of isotretinoin in sebo-supression. ${ }^{6,9,11}$

However, our bibliographical review did not identify any clinical essays performed with patients whose sebaceous hyperplasia had been treated with systemic isotretinoin.

Isotretinoin is known to be an inhibitor of the size and function of sebaceous glands. ${ }^{10}$ As it has been used for over two decades to treat acne, there exists vast knowledge about its administration and, especially, its side effects. ${ }^{12}$

In the few papers identified that discuss its use in sebaceous hyperplasia, isotretinoin was used in low doses on a small number of patients, who were generally monitored for only 2 months. ${ }^{4}$ This casts doubt over the lesion recurrence rate after the end of therapy mentioned in those studies.

In practice, it is known that decreasing the dosage of isotretinoin in treating acne can provoke a higher number of relapses, although some papers on

Received on 03.10.2013.

Approved by the Advisory Board and accepted for publication on 31.01.2014.

Work performed at the Dermoclínica - Dermatologia e Estética - Campinas (SP), Brazil.

Financial Support: FAPESP

Conflict of Interest: none.

Private clinic - Campinas (SP), Brazil.

Universidade Estadual de Campinas (UNICAMP) - Campinas (SP), Brazil.

Universidade Federal de São Paulo (UNIFESP) - São Paulo (SP), Brazil. 
dosage reduction to minimize side effects have reported low recurrence. ${ }^{13}$

\section{METHODS}

In this clinical trial, therapy entailed a monthly dosage similar to that normally used in acne treatment, though for a shorter duration. The protocol adopted was isotretinoin at a dosage of $1 \mathrm{mg} / \mathrm{kg} /$ day, for two months).

Firstly, 20 patients were selected for the clinical trial according to the following criteria:

Inclusion criteria: at least 18 years old, with clinical and histopathological diagnosis of sebaceous hyperplasia.

Exclusion criteria: under 18 years old, pregnant women or women at childbearing age who do not practice contraception, patients with dyslipidemia and/or altered liver function.

However, the number was decreased to 20 patients because some patients could not tolerate the side effects of mucosa and skin dryness and subsequently abandoned the treatment or reduced drug doses themselves. They were excluded from the group; some were monitored in parallel to the study, while others were lost during follow-up. Since results were very similar in all the individuals examined, there was no statistical requirement to increase the number, as this would merely have repeated the previously obtained results.

All selected patients signed an informed consent form, duly approved by the Research Ethics Committee at UNIFESP. There were no conflicts of interest in the study.

Patients' skin lesions were counted and photographed before and after treatment. They were monitored for two years after the end of treatment in order to evaluate the rate of recurring lesions and be photographed again. Five patients did not return after the two years for a recount of lesions. The side effects of the drug were also evaluated.

Changes in the number of lesions were examined, comparing numbers at the beginning of isotretinoin treatment and afterwards, using the Wilcoxon signed-rank test for comparison, since a decrease was observed in the number of lesions following use of the medication, in all patients $(n=20)$.

Variations were analyzed at two different points: after two months and after two years of isotretinoin treatment. In the two-year evaluation, we worked with $n=15$, since five patients did not return for monitoring. Throughout this assessment, we used the Wilcoxon signed-rank test, as patients were observed at three particular moments (the beginning plus the two other analysis points), and because the difference between the number of lesions at the moments analyzed did not approach a normal distribution (Gaussian). A confidence level of $\mathrm{p}<0.05$ was adopted.

We monitored patients for two years after the end of treatment in order to assess the rates of recurring lesions.

\section{RESULTS AND DISCUSSION}

The period of isotretinoin use was effective as regards the reduction in hyperplasia lesions, thus corroborating the literature reviewed, in which sub-doses of the medication were effectual at diminishing the size of the sabeceous gland. ${ }^{13}$

Our study has shown that $100 \%$ of the patients saw a significant decrease in the number of sebaceous hyperplasia lesions in response to treatment. Furthermore, note was taken of the fast therapeutic action of systemic isotretinoin (Figures 1 to 6).

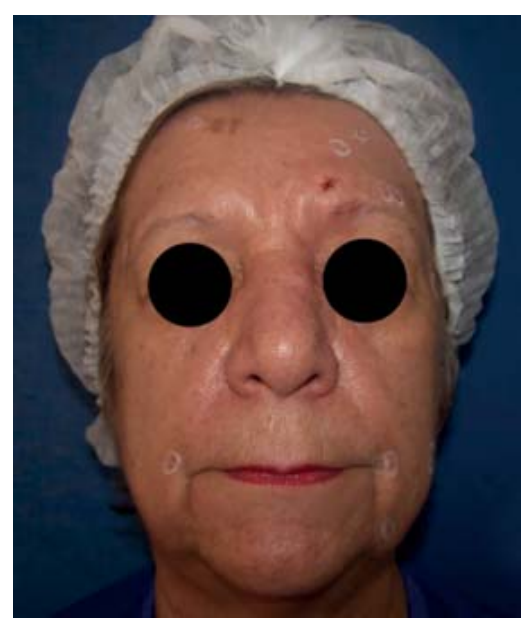

Figure 1:

Before treatment: 13 lesions

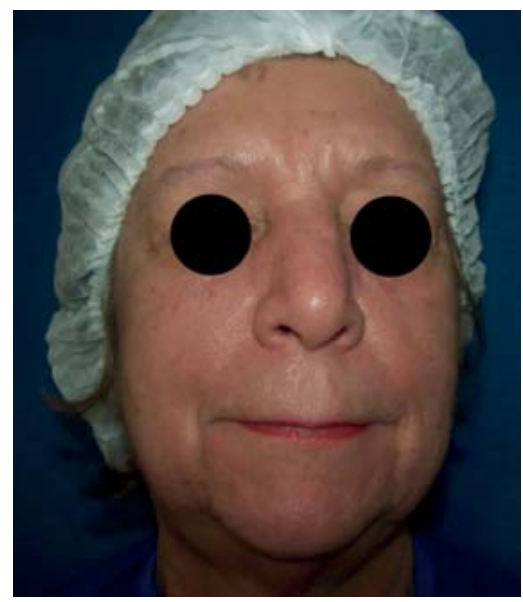

FIGURE 2:

Two months after treament: zero lesions 


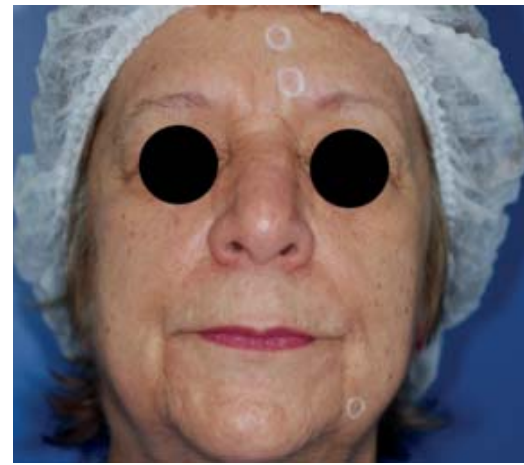

FIGURE 3:

Two years after treatment: 3 lesions

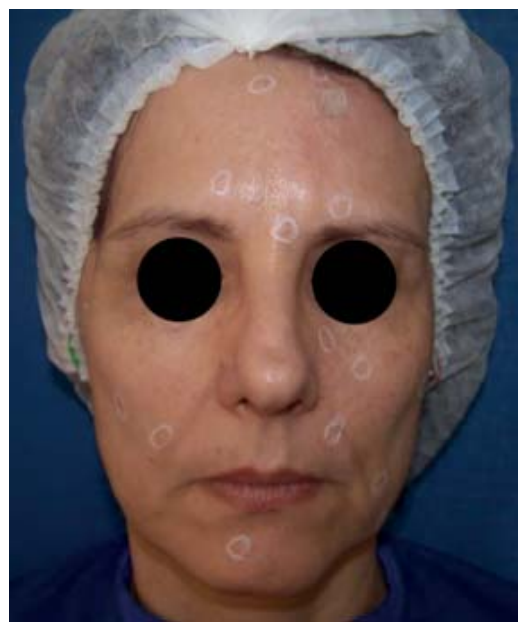

FIgURE 4:

Before treatment: 18 lesions

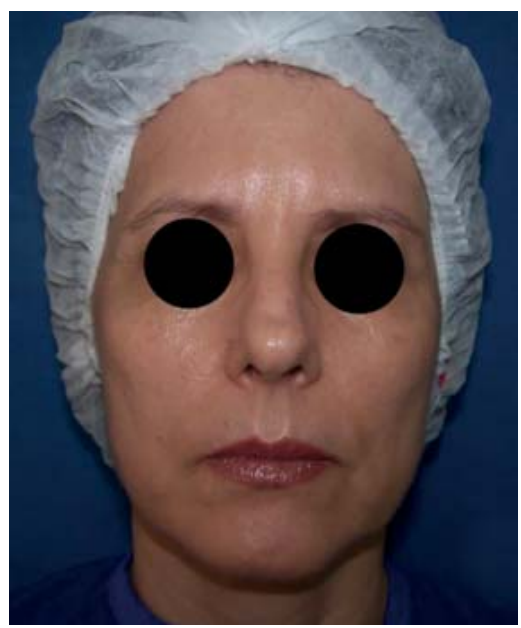

Figure 5:

Two months after treament: zero lesions.

The data analyzed demonstrated that, when evaluating the patients at the three stages of the study (before treatment, after two months of therapy and two years without it), there was a reduction in the average of 24 lesions (when monitoring first began) to 2 lesions (at two months of treatment). Importantly, all

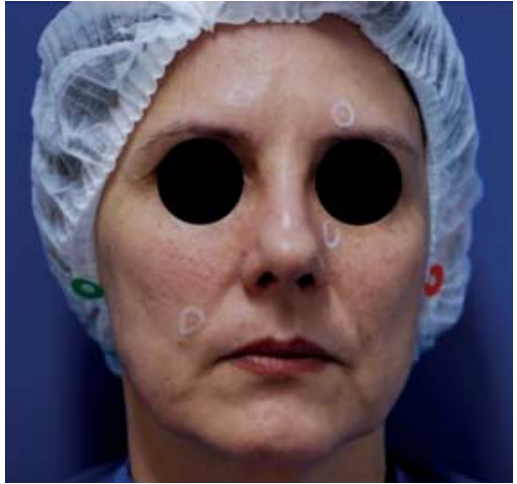

FIGURE 6:

Two years after treatment: 4 lesions

patients experienced a reduction in the number of lesions and even complete resolution in some cases.

When comparing results two years after the end of treatment, we noted few recurring hyperplasia lesions, with an average of 4 lesions. During the same periods, the median number of lesions decreased from 22 (i.e. $50 \%$ of the patients had over 22 lesions, while $50 \%$ had under 22) to zero, following two months' treatment. Equally, two years on from the end of medication use, there was an increase from the median of zero to three. This increase is statistically significant, but is of little clinical importance to most patients, since, despite recurrences in some patients, they still invariably had fewer lesions two years later than they did prior to initiating treatment (Table 1).

TABLE 1: Comparison of the number of lesions at the three stages of study

\begin{tabular}{lllll}
\hline Stage of Treatment & Average & Median & $\begin{array}{l}\text { Standard } \\
\text { Deviation }\end{array}$ & Test * \\
\hline $\begin{array}{l}\text { Beginning } \\
\text { After the treatment }\end{array}$ & 24 & 22 & 16 & $\mathrm{p}<0.05$ \\
(2 months) & & 0 & 3 & \\
\hline $\mathrm{p}=0.0001 ; \mathrm{n}=20$ & & & & \\
\hline
\end{tabular}

\begin{tabular}{lllll}
\hline Stage of Treatment & Average & Median & $\begin{array}{l}\text { Standard } \\
\text { Deviation }\end{array}$ & Test * \\
\hline $\begin{array}{l}\text { Beginning } \\
\text { After the treatment }\end{array}$ & 24 & 22 & 16 & $\mathrm{p}<0.05$ \\
$(2$ years) & & 3 & 5 & \\
$\mathrm{p}=0.0001 ; \mathrm{n}=15$ & & & \\
\hline
\end{tabular}

\begin{tabular}{lllll}
\hline Stage of Treatment & Average & Median & $\begin{array}{l}\text { Standard } \\
\text { Deviation }\end{array}$ & Test * \\
\hline $\begin{array}{l}\text { After the treatment } \\
\text { (2 months) }\end{array}$ & 2 & 0 & 3 & $\mathrm{p}<0.05$ \\
$\begin{array}{l}\text { After the treatment } \\
(2 \text { years })\end{array}$ & 4 & 3 & 5 & \\
\hline $\mathrm{p}=0.0078 ; \mathrm{n}=15$ & & & & \\
* Wilcoxon signed-rank test & & & &
\end{tabular}


NB.: There was evidence of a significant statistical difference between the number of lesions at the two moments evaluated.

Important note: One should remember that statistical significance must always be analyzed with reference to clinical significance. Potentially, there could be evidence of statistical difference and yet this difference could still be clinically insignificant.

As regards side effects, use of a recognized drug ensured safety for its administration. In particular, we observed dryness of the skin and mucosa, which were expected, given our experience with its use in acne therapy and the literature consulted about the drug. ${ }^{5,14}$ During the short treatment with isotretinoin, there was only one case where triglyceride levels rose, which was addressed with monthly serum dosages of circulating fats, leading to the return to basal levels one month after the end of the protocol, along with dietary reorientation.

Cosmetic improvement in skin through using the medication, involving a reduction in blemishes and softening of fine wrinkles, has been reported by most patients, in line with our experience and the literature reviewed..$^{15}$

Isotretinoin use at an age when pregnancy is no longer a priority nor even possible in many cases, minimized our concerns about the main side effect of isotretinoin (teratogenicity), although countermeasures must always be taken. ${ }^{5,12,16}$

It was surprising to observe the maintenance of the therapeutic result for two years after the end of the treatment, with few recurring lesions. Given the short length of treatment (two months), we expected a larger number of lesions after that period, since recurrence rates are reported to be around 19\%, even in prolonged acne treatment. ${ }^{17}$

Although we obtained $100 \%$ effectiveness regarding the decrease in lesions, it is important to highlight that new studies are necessary for better standardization of dosage and treatment length.

We propose new research protocols with the aim of revealing the minimum dosage necessary for hyperplasia resolution, as we believe that the dosage of $1 \mathrm{mg} / \mathrm{kg} /$ day for two months will not be necessary, since, during treatment, patients already showed a decrease in the number and size of lesions before completing the sixty days of proposed therapy. Some patients, who were subsequently excluded from this study, reduced the medication dosage themselves and achieved similar results to those who followed the protocol correctly.

Consequently, standardizing smaller doses promotes isotretinoin use in sebaceous hyperplasia treatment, due to the reduced costs and side effects, which are, as seen in literature, dosage-dependent., ${ }^{5,14,18-20}$

\section{CONCLUSION}

Ultimately, we were able to confirm that systemic isotretinoin use is an effective procedure in treating cutaneous sabeceous hyperplasia. 


\section{REFERENCES}

1. Bader RS, Scarborough DA. Surgical pearl: intralesional electrodesiccation of sebaceous hyperplasia. J Am Acad Dermatol. 2000;42:127-28.

2. González S, White WM, Rajadhyaksha M, Anderson RR, González E. Confocal imaging of sebaceous gland hyperplasia in vivo to assess efficacy and mechanism of pulsed dye laser treatment. Lasers Surg Med. 1999;25:8-12.

3. Gold MH, Bradshaw VL, Boring MM, Bridges TM, Biron JA, Lewis TL. Treatment of sebaceous gland hyperplasia by photodynamic therapy with 5 -aminolevulinic acid and a blue light source or intense pulsed light source. J Drugs Dermatol. 2004;3:S6-S9

4. Grekin RC, Ellis CN. Isotretinoin for the treatment of sebaceous hyperplasia. Cutis. 1984;34:90-2.

5. Orfanos CE, Zouboulis CC. Oral retinoids in the treatment of seborrhoea and acne. Dermatology. 1998;196:140-7.

6. Papakonstantinou E1, Aletras AJ, Glass E, Tsogas P, Dionyssopoulos A, Adjaye J, et al. Matrix metalloproteinases of epithelial origin in facial sebum of patients with acne and their regulation by isotretinoin. J Invest Dermatol. 2005 0ct;125:673-84.

7. Nelson AM, Zhao W, Gilliland KL, Zaenglein AL, Liu W, Thiboutot DM. Neutrophil gelatinase-associated lipocalin mediates 13-cis retinoic acid-induced apoptosis of human sebaceous gland cells. J Clin Invest. 2008;118:1468-78.

8. Torma H. Interaction of isotretinoin with endogenous retinoids. J Am Acad Dermatol. 2001;45:S143-9.

9. Trivedi NR, Cong Z, Nelson AM, Albert AJ, Rosamilia LL, Sivarajah S, et al. Peroxisome proliferator-activated receptors increase human sebum production. J Invest Dermatol. 2006;126:2002-9.

10. Hommel L, Geiger JM, Harms M, Saurat JH. Sebum excretion rate in subjects treated with oral all-trans-retinoic acid. Dermatology. 1996;193:127-30.

11. Zouboulis CC. Retinoids--which dermatological indications will benefit in the near future? Skin Pharmacol Appl Skin Physiol. 2001;14:303-15.

12. Meigel WN. How safe is oral isotretinoin? Dermatology. 1997;195:22-8.

13. Amichai B, Shemer A, Grunwald MH. Low-dose isotretinoin in the treatment of acne vulgaris. J Am Acad Dermatol. 2006 Apr;54(4):644-6.

14. Torrelo A, Pastor MA, Zambrano A. Severe acne infantum successfully treated with isotretinoin. Pediatr.Dermatol. 2005;22:357-59.

15. Hernandez-Perez E, Khawaja HA, Alvarez TY. Oral isotretinoin as part of the treatment of cutaneous aging. Dermatol Surg. 2000;26:649-52.

16. Saurat JH. Oral isotretinoin. Where now, where next? Dermatology. 1997:195:1-3

17. Ghalamkarpour F, Nasiri S. Isotretinoin in treatment of acne: its efficacy, side effects, and recurrence rate of disease. Arch Iran Med. 2006;9:228-30.

18. Kunynetz RA. A review of systemic retinoid therapy for acne and related conditions. Skin Therapy Lett. 2004;9:1-4.

19. Wysowski DK, Swann J, Vega A. Use of isotretinoin (Accutane) in the United States: rapid increase from 1992 through 2000. J Am Acad Dermatol. 2002:46:505-9.

20. Zouboulis CC, Xia L, Akamatsu H, Seltmann H, Fritsch M, Hornemann S, et al. The human sebocyte culture model provides new insights into development and management of seborrhoea and acne. Dermatology. 1998;196:21-31.

\author{
MAILING ADDRESS: \\ Sandra Tagliolatto \\ Rua Luzitana -740 - $4^{\circ}$ andar - Bosque \\ 13015121 -Campinas - SP \\ Brazil \\ E-mail:dermoclinica@dermoclinica.med.br
}

How to cite this article: Tagliolatto S, Santos Neto OO, Alchorne MAM, Enokihara MY. Sebaceous hyperplasia: systemic treatment with isotretinoin. An Bras Dermatol. 2015;90(2):211-5. 